\title{
AUC-Based Linear Combination of Dichotomizers
}

\author{
Claudio Marrocco, Mario Molinara, and Francesco Tortorella \\ Dipartimento di Automazione, Elettromagnetismo, \\ Ingegneria dell'Informazione e Matematica Industriale \\ Università degli Studi di Cassino \\ 03043 Cassino (FR), Italy \\ \{c.marrocco, m.molinara, tortorella\}@unicas.it
}

\begin{abstract}
The combination of classifiers is an established technique to improve the classification performance. The combination rules proposed up to now generally try to decrease the classification error rate, which is a performance measure not suitable in many real situations and particularly when dealing with two class problems. In this case, a good alternative is given by the Area under the Receiver Operating Characteristic curve (AUC). This paper presents a method for the linear combination of two-class classifiers aimed at maximizing the AUC. The effectiveness of the approach has been confirmed by the tests performed on standard datasets.
\end{abstract}

\section{Introduction}

Linear combination is a widely diffused technique for combining multiple classifiers. The main reasons are both its simplicity and effectiveness [1]. This can be particularly useful in two-class problems that require highly discriminating classifiers. To this aim, linear combiners are generally built with the aim of minimizing the classification error. However, the considered applications frequently involve cost matrices and class distributions both strongly asymmetric and dynamic and in such cases the overall error rate, usually employed as a reference performance measure in classification problems, is not a suitable metric to evaluate the quality of the classifier [2]. An important tool to correctly analyse the performance of the classifier under different class and cost distributions is given by the Receiver Operating Characteristic (ROC) curve that provides a description of the performance of the dichotomizer at different operating points independently of the prior probabilities of the two classes. Moreover, the geometrical properties of the ROC curve can be profitably used to optimise the performance of a dichotomizer with reference to various metrics and classification requirements. In this case, to compare different dichotomizers it could be useful to employ a single value measure that describes the quality of the classifier. To this aim, the most widely used single measure is the Area Under the ROC Curve (AUC) that represents a more effective and discriminating performance measure than the accuracy to evaluate the quality of dichotomizers [3].

In this paper, focusing on the simplest and most widely used implementation of linear combiners, which consists of assigning a nonnegative weight to each individual classifier, we propose a method to directly optimise the AUC. In fact, we consider the linear combination of several classifiers and propose a method to achieve the optimal weight vector of the combination. To this aim, an analysis of the dependence of the 
AUC of the linear combiner on the weighting is presented for two classifiers and a greedy approach to extend the combination rule to several classifiers is proposed together with the results of experiments performed on standard datasets that confirmed the effectiveness of the approach.

The rest of the paper is organized as follows: in the next section we present a short description of the AUC measure while section 3 shows the proposed method for two and $\mathrm{N}>2$ classifiers. Then the obtained experimental results are reported and in the last section some conclusions and possible future developments are drawn.

\section{AUC of a Dichotomizer}

In two-class classification problems, the goal is to build a dichotomizer $f(z)$ (i.e. a two-class classifier) which assigns a pattern $z$ coming from an instance space $S$ to one of two mutually exclusive classes that can be generically called Positive $(P)$ class and Negative $(N)$ class. Without loss of generality, let us assume that $f(x)$ is in the range $(-\infty,+\infty)$ and provides a confidence degree that the sample belongs to one of the two classes, e.g. the class $P$. The sample should be consequently assigned to the class $N$ if $f(x) \rightarrow-\infty$ and to the class $P$ if $f(x) \rightarrow+\infty$; actually the output of the dichotomizer is compared with a threshold $t$ to decide which is the class the sample should be assigned to. In order to evaluate performance of $f($.$) , we can consider the outputs$ provided by the trained classifier on a set $S$ containing $n_{+}$positive samples and $n$. negative samples $S=\left\{\mathrm{p}_{i} \in P, i=1 \ldots n_{+}\right\} \cup\left\{\mathrm{n}_{j} \in N, j=1 \ldots n_{-}\right\}$and build the empirical ROC curve [4], whose points $(F P R(t), T P R(t))$ are given by:

$$
\operatorname{TPR}(t)=\frac{1}{n_{+}} \sum_{i=1}^{n_{+}} \Pi\left(f\left(\mathrm{p}_{i}\right) \geq t\right) \quad F P R(t)=\frac{1}{n_{-}} \sum_{j=1}^{n_{-}} \Pi\left(f\left(\mathrm{n}_{j}\right) \geq t\right)
$$

where $t$ is in the range $(-\infty,+\infty)$ and $\Pi($.$) is a predicate which is 1$ when the argument is true and 0 otherwise. Based on the ROC curve, a widely used single measure is the Area Under the ROC Curve (AUC), which intuitively provides an estimate of the quality of the dichotomizer ( $\mathrm{AUC}=0.5$ for a non discriminating dichotomizer, $\mathrm{AUC}=1$ for a perfectly discriminating dichotomizer). The AUC of the dichotomizer $f($.) could be easily evaluated by numerically integrating the corresponding ROC. However, there is a useful equivalence [5] between the AUC of a dichotomizer and the Wilcoxon-Mann-Whitney (WMW) statistic which is defined as:

$$
\frac{\sum_{i=1}^{n_{+}} \sum_{j=1}^{n_{-}} I\left(f\left(\mathrm{p}_{i}\right), f\left(\mathrm{n}_{j}\right)\right)}{n_{+} \cdot n_{-}}
$$

where $I(x, y)$ returns 1 if $x>y, 0.5$ if $x=y$ and 0 if $x<y$. In this way, it is possible to evaluate the AUC of $f$ directly through (1) without explicitly plotting the ROC curve and estimating the area with a numerical integration.

Thanks to this equivalence, the AUC has a useful physical meaning: if we consider the outputs $f\left(x_{P}\right)$ and $f\left(x_{N}\right)$ provided by the dichotomizer on two samples $z_{P}$ and $z_{N}$ randomly extracted from $P$ and $N$, it can be demonstrated [6] that the WMW statistic in (1), and thus the AUC, provides an unbiased estimate of the probability 
$P\left(f\left(z_{P}\right)>f\left(z_{N}\right)\right)$. In other words, the AUC of a dichotomizer measures the probability of correct pair-wise ranking [7] and thus evaluates the performance of the classifier considered as a ranker.

\section{AUC-Based Linear Combination of Dichotomizers}

The purpose of the method we are going to introduce is to construct a linear combination of dichotomizers aimed at maximizing the AUC of the resulting classification system. We focus first on the combination of two dichotomizers and then extend to $\mathrm{N}>2$ dichotomizers.

\subsection{Linear Combination of Two Dichotomizers}

Let $S$ be a set of samples defined as above. Let us consider two dichotomizers $f_{0}$ and $f_{1}$ whose outputs on positive and negative samples are:

$$
x_{i}^{0}=f_{0}\left(\mathrm{p}_{i}\right) \quad y_{j}^{0}=f_{0}\left(\mathrm{n}_{j}\right) \quad x_{i}^{1}=f_{1}\left(\mathrm{p}_{i}\right) \quad y_{j}^{1}=f_{1}\left(\mathrm{n}_{j}\right)
$$

The AUC's for the two dichotomizers evaluated according to the WMW statistic are:

$$
\mathrm{AUC}_{0}=\frac{\sum_{i=1}^{n_{+}} \sum_{j=1}^{n_{-}} I\left(x_{i}^{0}, y_{j}^{0}\right)}{n_{+} \cdot n_{-}} \quad \mathrm{AUC}_{1}=\frac{\sum_{i=1}^{n_{+}} \sum_{j=1}^{n_{-}} I\left(x_{i}^{1}, y_{j}^{1}\right)}{n_{+} \cdot n_{-}}
$$

Let us now consider a linear combination of $f_{0}$ and $f_{1}$. Without any loss of generality, the resulting classifier can be represented by:

$$
f_{l c}=f_{0}+\alpha \cdot f_{1}
$$

where $\alpha$ is the relative weight of $f_{1}$ with respect to $f_{0}$. The outputs of $f_{l c}$ to $\mathrm{p}_{i}$ and $\mathrm{n}_{j}$ will be consequently:

$$
\xi_{i}=f_{l c}\left(\mathrm{p}_{i}\right)=x_{i}^{0}+\alpha \cdot x_{i}^{1} \quad \eta_{j}=f_{l c}\left(\mathrm{n}_{j}\right)=y_{j}^{0}+\alpha \cdot y_{j}^{1}
$$

According to the WMW statistic, the AUC of $f_{l c}$ is given by:

$$
\mathrm{AUC}_{l c}=\frac{\sum_{i=1}^{n_{+}} \sum_{j=1}^{n_{-}} I\left(\xi_{i}, \eta_{j}\right)}{n_{+} \cdot n_{-}}
$$

and depends on the value of $\alpha$. Therefore the optimal choice for the weight is the value maximizing $\mathrm{AUC}_{\mathrm{lc}}$. To this aim, let us analyze the term $I\left(\xi_{i}, \eta_{j}\right)$ and study how it depends on the values of $I\left(x_{i}^{0}, y_{j}^{0}\right)$ and $I\left(x_{i}^{1}, y_{j}^{1}\right)$; for the following analysis we consider a tie as an error and thus we group together the cases $I(x, y)=0.5$ and $I(x, y)=0$. With this assumption, the set of all the pairs on which $\mathrm{AUC}_{l c}$ is evaluated can be split in four subsets $S_{00}, S_{11}, S_{01}, S_{10}$, where $S_{u v}$ is defined as:

$$
S_{u v}=\left\{(i, j) \mid I\left(x_{i}^{0}, y_{j}^{0}\right)=u \text { and } I\left(x_{i}^{1}, y_{j}^{1}\right)=v\right\}
$$


Moreover, let $C_{u v}$ denote the number of pairs container in the set $S_{u v}$ : it can be simply verified that the expression for $\mathrm{AUC}_{l c}$ can be written as:

$$
\mathrm{AUC}_{l c}=\frac{1}{n_{+} \cdot n_{-}}\left[\sum_{(i, j) \in S_{00}} I\left(\xi_{i}, \eta_{j}\right)+\sum_{(i, j) \in S_{11}} I\left(\xi_{i}, \eta_{j}\right)+\sum_{(i, j) \in S_{10} \cup S_{01}} I\left(\xi_{i}, \eta_{j}\right)\right]=\frac{1}{n_{+} \cdot n_{-}}\left[0+C_{11}+v(\alpha)\right]
$$

In other words, while the pairs on which both dichotomizers are wrong do not contribute to $\mathrm{AUC}_{l c}$ and the pairs correctly ranked by both the dichotomizers give a contribution independent of the value of $\alpha$, the dependence of $\mathrm{AUC}_{l c}$ on $\alpha$ is limited to the set of pairs on which the dichotomizers disagree. Therefore, the larger the set $S_{10} \cup S_{01}$ (i.e., the higher the disagreement between $f_{0}$ and $f_{1}$ ), the higher the value of $\mathrm{AUC}_{l c}$ which, in principle, can be obtained. Taking into account eq. (9), the optimal value for $\alpha$ can be defined as $\alpha_{\text {opt }}=\arg \max v(\alpha)$. In order to find such value let us make explicit the dependence of $I\left(\xi_{i}, \eta_{j}\right)$ on $\alpha$, to this aim, recall that the indicator function is not null only if $\xi_{i}>\eta_{j}$, i.e. if:

$$
\left(x_{i}^{0}-y_{j}^{0}\right)+\alpha \cdot\left(x_{i}^{1}-y_{j}^{1}\right)>0
$$

To simplify the following calculations, let us call Score Difference Ratio (SDR) the quantity $-\frac{x_{i}^{0}-y_{j}^{0}}{x_{i}^{1}-y_{j}^{1}}$ and denote it with $\Delta_{1}^{0}(i, j)$; for pairs $(i, j)$ belonging to $S_{01}$ or $S_{10}$ this value is positive because in both cases the differences have opposite signs. The condition (10) leads to different constraints on $\alpha$ depending on which of the two sets $S_{01}$, $S_{10}$ we consider. In particular we obtain:

$$
\alpha<\Delta_{1}^{0}(i, j) \text { if }(i, j) \in S_{10} \quad \alpha>\Delta_{1}^{0}(i, j) \text { if }(i, j) \in S_{01}
$$

If such conditions were verified for each pair $(i, j) \in S_{10} \cup S_{01}$, we would obtain the max value allowable for $v(\alpha)$, i.e. $C_{10}+C_{01}$, but in general this cannot be obtained since the distributions of the SDRs coming from the sets $S_{10}$ and $S_{01}$ are not separated. In any case, $\alpha_{o p t}$ has to be found by maximizing the number of the pairs satisfying eq. (10). To this aim, let us evaluate how many pairs of each set are correctly ranked for a given value $\alpha$ for the weight of the linear combination. In order to simplify the notation in the following analysis, let us disregard the dependence on the particular samples in the SDRs and denote with $\delta_{h}^{01}$ with $h=1, \ldots, C_{01}$ the SDR value of the $h$-th pair contained in $S_{01}$; in a similar way, let $\delta_{k}^{10}$ with $k=1, \ldots, C_{10}$ indicate the SDR value of the $k$-th pair contained in $S_{10}$. With this notation, we define the Correctly Ranked Rate on $S_{01}, C R R_{01}(\alpha)$, and the Wrongly Ranked Rate on $S_{01}, W R R_{01}(\alpha)$, as:

$$
C R R_{01}(\alpha)=\frac{\#\left\{\delta_{h}^{01}<\alpha, h=1 \ldots C_{01}\right\}}{C_{01}} \quad W_{R} R_{01}(\alpha)=\frac{\#\left\{\delta_{h}^{01} \geq \alpha, h=1 \ldots C_{01}\right\}}{C_{01}}
$$

Both indices are in the range [0,1] and are not independent since they sum up to 1 . In a similar way, it is possible to evaluate the same indices on the set $S_{10}$ :

$$
C R R_{10}(\alpha)=\frac{\#\left\{\delta_{k}^{10}>\alpha, k=1 \ldots C_{10}\right\}}{C_{10}} \quad W R R_{10}(\alpha)=\frac{\#\left\{\delta_{k}^{10} \geq \alpha, k=1 \ldots C_{10}\right\}}{C_{10}}
$$


Since for each set the indices are dependent on each other, it is sufficient to know only one index for each set in order to have the corresponding value for $v(\alpha)$. A possible choice could be to consider only $W R R_{01}$ and $C R R_{10}$ and to represent them as coordinates in a plane: in this way, the values produced by a particular $\alpha$ individuate a point in the unit square whose corners are the points $(0,0),(1,0),(0,1)$ and $(1,1)$. When the value of the weight $\alpha$ varies between 0 and $\infty$ the quantities $W R R_{01}$ and $C R R_{10}$ vary accordingly, thus drawing a curve running from $(1,1)$ to $(0,0)$. We call it Difference Ratio Operating Characteristic curve (DROC curve).

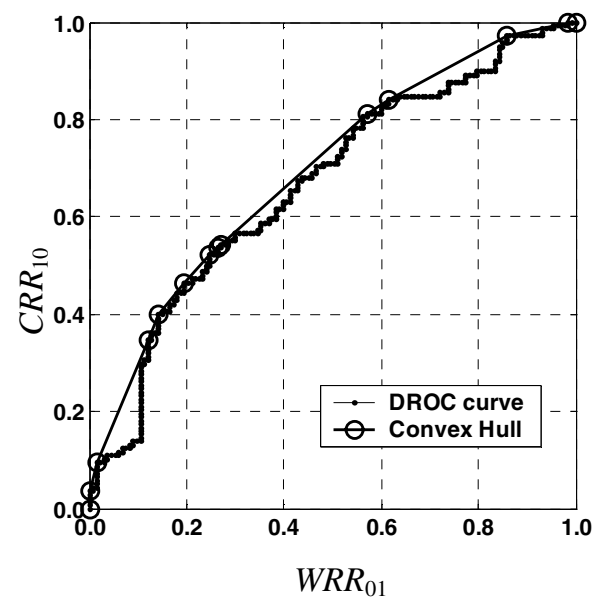

Fig. 1. A DROC curve together with the related convex hull

The DROC can be profitably used also for selecting the optimal value of the weight $\alpha_{o p t}$. To this aim, let us point out that the quantity to be maximized in eq. (9) can be written as:

$$
v(\alpha)=C_{10} \cdot C R R_{10}(\alpha)+C_{01} \cdot\left(1-W R R_{01}(\alpha)\right)
$$

In the case the DROC curve is defined by means of a finite number of experimental points connected with straight lines (similar to the curve shown in fig. 1), it can be demonstrated that $\alpha_{o p t}$ can be determined by locating the point where a line with slope $m=\frac{C_{01}}{C_{10}}$, moving down from above, touches the DROC curve and taking the corresponding value of $\alpha$. In particular, such point lies on the DROC Convex Hull, i.e. the smallest convex set containing the points of the DROC curve.

\subsection{Linear Combination of N>2 Dichotomizers}

Let us now consider the linear combination of several (say N) classifiers:

$$
f_{l c}=\alpha_{0} f_{0}+\alpha_{1} f_{1}+\ldots+\alpha_{\mathrm{N}-1} f_{\mathrm{N}-1}
$$


In order to find the optimal weight vector $\alpha_{\mathrm{opt}}=\left(\begin{array}{llll}\alpha_{0} & \alpha_{1} & \cdots & \alpha_{\mathrm{N}-1}\end{array}\right)$ that maximizes the AUC associated with $f_{l c}$, we should extend the algorithm proposed in the previous section. Unfortunately, eq. (11) cannot be generalized to $\mathrm{N}>2$ dichotomizers in such a way that the maximization of the resulting AUC is computationally feasible. For this reason, we adopted a suboptimal algorithm that approximates the solution using a greedy approach. Hence, rather than considering every possible combination in its entirety, we iteratively find the optimal weight of the linear combination of two dichotomizers so as to evaluate all the combination weights in N-1 steps. In this context an important role is played by the order of combination, i.e. which pair of classifiers should be combined first. Since we know from previous sections that the greater is the diversity among the classifiers to be combined the greater is the improvement to the performance of the base classifiers which could be gained, we choose the pair that exhibits the maximum disagreement coefficient. Once the weight has been computed, the two dichotomizers are replaced by their combination and thus the dichotomizers to be combined decrease from $\mathrm{N}$ to $\mathrm{N}-1$. At this point, we have to evaluate the disagreement between the new classifier and the other classifiers. It is worth noting that, for this step, it is not necessary to compute the output of the new classifier, since its score differences can be directly evaluated as the weighted sum (with the same weight estimated for the combination) of the score differences of the combined classifiers. These steps are repeated until all the dichotomizers have been combined: in each iteration it is chosen the pair of dichotomizers with the highest disagreement coefficient. At the end, we obtain the weight vector $\alpha_{\text {opt }}$. It is worth noting that also in this case one of the weights of the vector will be equal to 1 , but this will not imply any loss of generality.

\section{Experimental Results}

In order to evaluate the performance of the proposed method, it has been tested on three datasets publicly available at the UCI Machine Learning Repository [8]. All of them have two classes and a variable number of numerical input features. The features were previously rescaled so as to have zero mean and unit standard deviation. To avoid any bias in the comparison, 10 runs of a multiple hold out procedure have been performed on all data sets. In each run, the dataset has been parted into three sets: a training set to train the classifier, a validation set to estimate the optimal weight vector and a test set to assess the reliability of the proposed method. More details for each dataset are given in table 1.

Table 1.

\begin{tabular}{|c|c|c|c|c|c|c|c|}
\hline Datasets & $\begin{array}{c}\# \\
\text { Features }\end{array}$ & $\begin{array}{c}\# \\
\text { Samples }\end{array}$ & $\%$ Pos. & $\%$ Neg. & $\begin{array}{c}\text { Train. } \\
\text { Set }\end{array}$ & $\begin{array}{c}\text { Valid. } \\
\text { Set }\end{array}$ & $\begin{array}{c}\text { Test } \\
\text { Set }\end{array}$ \\
\hline $\begin{array}{c}\text { Australian Credit } \\
\text { Approval }\end{array}$ & 14 & 690 & 44.5 & 55.5 & 483 & 103 & 104 \\
\hline $\begin{array}{c}\text { Contraceptive } \\
\text { Method Choice }\end{array}$ & 9 & 1473 & 57.3 & 42.7 & 1031 & 221 & 221 \\
\hline $\begin{array}{c}\text { Pima Indian } \\
\text { Diabetes }\end{array}$ & 8 & 768 & 34.9 & 65.1 & 538 & 115 & 115 \\
\hline
\end{tabular}


The employed dichotomizers are Support Vector Machines (SVM) and Multi Layer Perceptrons (MLP). The SV-based classifiers have been implemented by means of SVMlight tool [9] while for the MLPs we have used the NODElib library [10]. Six different kernels have been used for the SVMs (linear, polynomial of degree 2 and 3, gaussian with $\left.\sigma^{2}=5,2,1\right)$ while for the MLPs we have considered three classifiers with different numbers of units $(2,4,6)$ in the hidden layer, all trained for 10000 epochs using the back propagation algorithm with a learning rate of 0.01 .

For the sake of comparison, we have also considered another method which evaluates the weight vector maximizing the AUC by employing a bound constrained global optimization algorithm, called Multilevel Coordinate Search (MCS) [11]. It is based on a multilevel coordinate search that balances global and local search; the local search is done via sequential quadratic programming and it is not exhaustive. Of course, the aim here is not to provide another algorithm to construct the optimal combination, but to obtain a reliable estimate of the weight vector maximizing the AUC on the validation set (even though with a computationally expensive algorithm) with which to compare the results provided by our method.

In order to choose a set of combinations of dichotomizers significant for the comparison, we have preliminarily ordered the single classifiers according to their mean AUC evaluated on the validation set and then we have built 7 different groups by putting together the $K$ best classifiers (with $K$ ranging from 2 to 8) and other 7 groups collecting the $K$ worst classifiers. For each of these groups we have built two linear combinations, the first obtained with our DROC-based method while the second employs the weight vector estimated by means of the MCS algorithm.
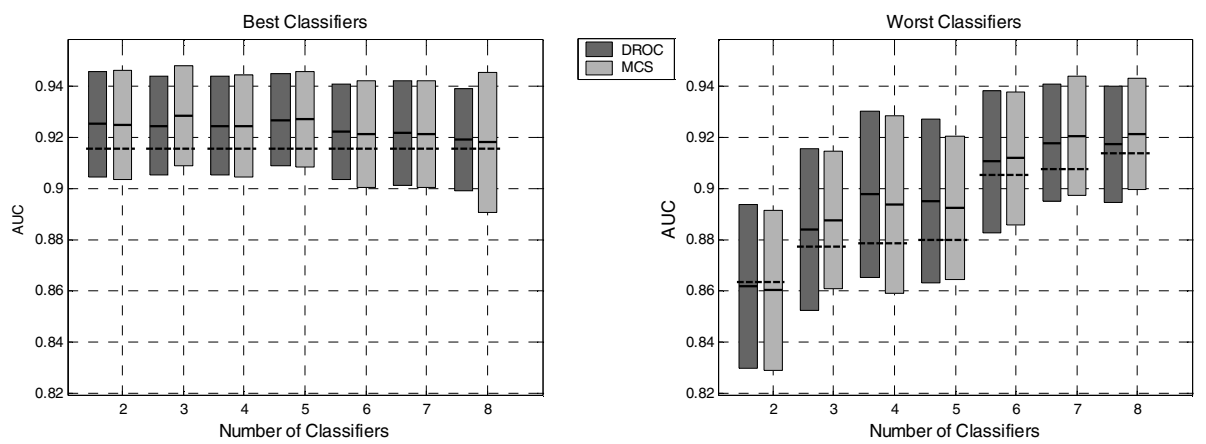

Fig. 2. The results on the Australian data set

In figures 1-3 we report the results in terms of AUC obtained on the test set for each data set. In particular, we show the mean value and the error bars relative to each method together with the performances of the best single classifier of the group (dashed line). From these results we can see that the DROC based method is actually able to determine the best weight vector: in fact the two methods provide quite the same performance (both in terms of average AUC and standard deviation) in all the examined cases. Obviously, they are both more proficient when working with the weaker classifiers. 

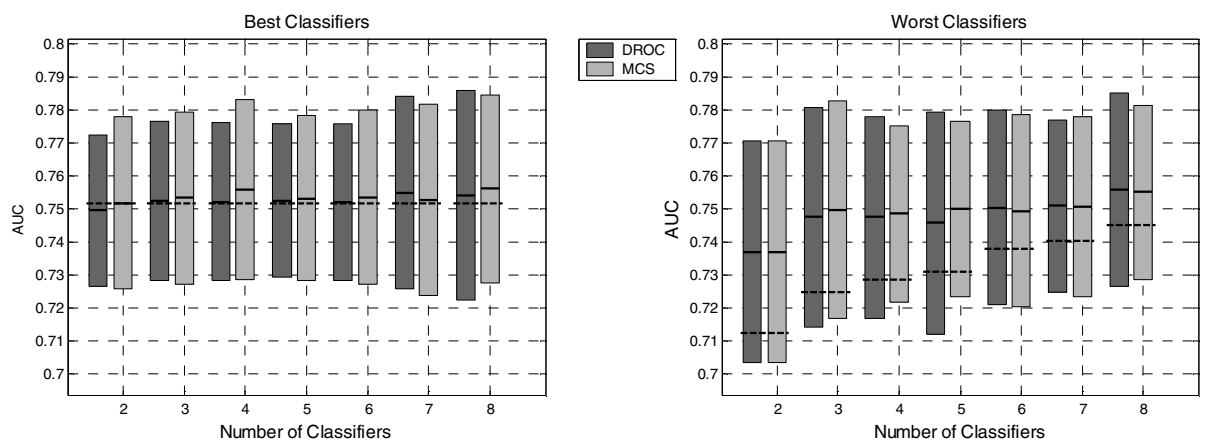

Fig. 3. The results on the Contraceptive data set
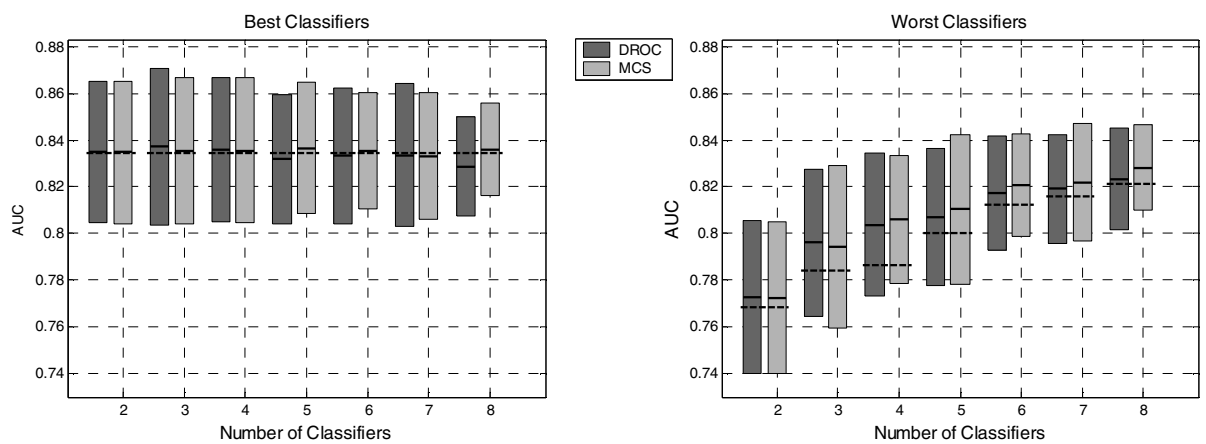

Fig. 4. The results on the Pima data set

\section{Conclusions}

In this paper we have introduced a method for the linear combination of dichotomizers with the aim of maximizing the AUC of the resulting classification system. The method is based on an analysis of the dependence of the AUC of the linear combiner on the weight $\alpha$ in the case of two dichotomizers and is extended to the general case through a greedy approach. The experiments have demonstrated that the algorithm actually allows the optimal value of the weight vector to be found. The future researches on this topic will consider the comparison with other linear combination methods (simple and weighted average) as well as a more thorough analysis of the direct relation found between the performance improvement attainable with the combination and the measure of the disagreement existing between the dichotomizers.

\section{References}

1. Fumera G., Roli F., 2005. A Theoretical and Experimental Analysis of Linear Combiners for Multiple Classifier Systems. IEEE Trans. on Patt. Anal. and Mach. Intell., vol. 27, no. 6, 942-956.

2. Provost, F., Fawcett, T., Kohavi, R., 1998. The Case against Accuracy Estimation for Comparing Induction Algorithms. Proc. ICML-1998, Morgan Kaufmann, 445-453. 
3. Huang, J., Ling, C.X., 2005. Using AUC and Accuracy in Evaluating Learning Algorithms. IEEE Transactions on Knowledge and Data Engineering 17, 299-310.

4. A. Webb, 2002. Statistical pattern Recognition, $2^{\text {nd }}$ ed., Wiley, USA.

5. Pepe, M., 2003. The Statistical Evaluation of Medical Tests for Classification and Prediction. Oxford University Press, Oxford, UK.

6. Lehmann, E.L., 1975, Nonparametrics. Statistical Methods Based on Ranks, Holden-Day, S. Francisco, USA.

7. Hanley J.A., McNeil B.J., 1982. The Meaning and the Use of the Area under a Receiver Operating Characteristic Curve. Radiology 143, 29-36.

8. Blake, C., Keogh, E., Merz, C.J.: UCI Repository of Machine Learning Databases. (1998) [www.ics.uci.edu/ mlearn/MLRepository.html]

9. Joachims, T.: Making Large-Scale SVM Learning Practical, in Schölkopf, B., Burges, C.J.C., Smola, A.J., eds., Advances in Kernel Methods, MIT Press (1999) 169-184.

10. Flake, G.W., Pearlmuter, B.A., 2000. Differentiating Functions of the Jacobian with Respect to the Weights. In S. A. Solla, T. K. Leen, and K. Müller, eds., Advances in Neural Information Processing Systems 12, The MIT Press.

11. Huyer, W., A. Neumaier, A., 1999. Global optimization by multilevel coordinate search, J. Global Optimization 14, 331-355. 